\title{
A generalization to almost balancing and cobalancing numbers using triangular numbers
}

\author{
S. G. Rayaguru ${ }^{1}$ and G. K. Panda ${ }^{2}$ \\ ${ }^{1}$ Department of Mathematics, National Institute of Technology \\ Rourkela, India \\ e-mail: saigopalrs@gmail.com \\ ${ }^{2}$ Department of Mathematics, National Institute of Technology \\ Rourkela, India \\ e-mail: gkpanda_niterediffmail.com
}

\begin{abstract}
A generalization of almost balancing numbers is studied using triangular numbers as the difference between the left and right hand sides of the defining equation of balancing numbers. In case of almost balancing numbers, this difference is kept 1 , which is the first triangular number. Some specific representations of these numbers in terms of balancing and balancing related numbers are established and few more results with triangular, square triangular, balancing and balancing related numbers are also studied so as to generalize the identities obtained by A. Tekcan.
\end{abstract}

Keywords: Balancing numbers, Cobalancing numbers, Almost balancing numbers, Lucasbalancing numbers, Lucas-cobalancing numbers, Triangular numbers.

2010 Mathematics Subject Classification: 11B39, 11B38.

\section{Introduction}

A natural number $n$ is called a balancing number [1] or cobalancing number [14] accordingly, as

$$
1+2+\cdots+(n-1)=(n+1)+(n+2)+\cdots+(n+r)
$$

or

$$
1+2+\cdots+n=(n+1)+(n+2)+\cdots+(n+r)
$$


holds for some natural number $r$. This $r$ is called a balancer associated with $n$ in case the former equation holds and is called a cobalancer associated with $n$ if the later equation holds. One can check that $6,35,204$ are balancing numbers with corresponding balancers 2, 14, 84 while, $2,14,84$ are cobalancing numbers with cobalancers $1,6,35$ respectively. If $n$ is a balancing number, then $8 n^{2}+1$ is a perfect square [1] and if $n$ is a cobalancing number, then $8 n^{2}+8 n+1$ is a perfect square [14].

The $k^{\text {th }}$ balancing and cobalancing numbers are denoted by $B_{k}$ and $b_{k}$ respectively. Furthermore, $C_{k}=\sqrt{8 B_{k}^{2}+1}$ and $c_{k}=\sqrt{8 b_{k}^{2}+8 b_{k}+1}$ are called the $k^{\text {th }}$ Lucas-balancing and Lucas-cobalancing numbers respectively (see $[12,14]$ ). The balancing numbers can be calculated recursively as $B_{n+1}=6 B_{n}-B_{n-1}$ with initial terms $B_{0}=0$ and $B_{1}=1$. The Lucas-balancing and Lucas-cobalancing numbers satisfy recurrence relations identical with balancing numbers with initial terms $C_{0}=1, C_{1}=3, c_{0}=-1, c_{1}=1$. However, the cobalancing numbers satisfy $b_{n+1}=6 b_{n}-b_{n-1}+2$ with $b_{0}=b_{1}=0$. The Binet forms of these numbers are given by

$$
B_{n}=\frac{\alpha^{2 n}-\beta^{2 n}}{4 \sqrt{2}}, C_{n}=\frac{\alpha^{2 n}+\beta^{2 n}}{2}, b_{n}=\frac{\alpha^{2 n-1}-\beta^{2 n-1}}{4 \sqrt{2}}-\frac{1}{2}, c_{n}=\frac{\alpha^{2 n-1}+\beta^{2 n-1}}{2} .
$$

where $\alpha=1+\sqrt{2}$ and $\beta=1-\sqrt{2}$. These numbers are very much interrelated and appear in several fascinating identities (see $[1,5,6,8,10,12,15,17,20]$ ).

If $B$ is a balancing number then $B^{2}$ is a triangular number and for a cobalancing number $b$, $b^{2}+b$ is a triangular number. The balancers and the cobalancers are also related to the triangular numbers in numerous ways. It is worth mentioning that the $k$-th triangular number is denoted by $T_{k}$ and is equal to $\frac{k(k+1)}{2}$.

Kovács, Liptai and Olajos [7] extended the concept of balancing numbers by defining $(a, b)$-balancing numbers. For coprime integers $a>0$ and $b \geq 0$, they called $a n+b$ an $(a, b)$-balancing number if the Diophantine equation

$$
(a+b)+\cdots+(a(n-1)+b)=(a(n+1)+b)+\cdots+(a(n+r)+b)
$$

holds for some positive integer $r$.

In [2], Dash, Ota and Dash defined the $t$-balancing numbers $n$ and $t$-balancers $r$ as solutions of the Diophantine equation

$$
1+2+\cdots+n=(n+1+t)+(n+2+t)+\cdots+(n+r+t) ; t \geq 2
$$

and some properties of these numbers have been studied in [19].

Rout and Panda [18] generalized the concept of balancing numbers and introduced gap balancing numbers. If $k$ is odd, they call a natural number $n$ a $k$-gap balancing number if

$$
1+2+\cdots+\left(n-\frac{k+1}{2}\right)=\left(n+\frac{k+1}{2}\right)+\left(n+\frac{k+3}{2}\right)+\cdots+(n+r)
$$

for some natural number $r$, which they call a $k$-gap balancer corresponding to $n$, while for $k$ even, if

$$
1+2+\cdots+\left(n-\frac{k}{2}\right)=\left(n+\frac{k}{2}+1\right)+\left(n+\frac{k}{2}+2\right)+\cdots+(n+r)
$$

for some natural numbers $n$ and $r$, then they call $2 n+1$ a $k$-gap balancing number and $r$ a $k$-gap balancer. 
Panda and Panda [13] defined almost balancing numbers as the values of $n$ satisfying the Diophantine equations

$$
|(n+1)+(n+2)+\cdots+(n+r)-[1+2+\cdots+(n-1)]|=1
$$

for some $r$, which they called an almost balancer corresponding to $n$. Furthermore, $n$ is called an almost cobalancing number with almost cobalancer $r$, if

$$
|(n+1)+(n+2)+\cdots+(n+r)-[1+2+\cdots+n]|=1
$$

(see [16]). Davala and Panda [3,4] generalized the concept of almost balancing and cobalancing numbers by defining $D$-subbalancing and $D$-supercobalancing numbers $n$ as solutions of the equations

$$
1+2+\cdots+(n-1)+D=(n+1)+(n+2)+\cdots+(n+r)
$$

and

$$
1+2+\cdots+n=(n+1)+(n+2)+\cdots+(n+r)+D
$$

respectively, where $D$ is any fixed positive integer. In (2), $r$ is called a $D$-subbalancer while in (3), $r$ is called a $D$-supercobalancer. They proved the existence of at least two classes of $B_{k}$-supercobalancing and $b_{k}$-subbalancing numbers. Motivated by this idea, we devote this paper to explore the existence of subbalancing, superbalancing, subcobalancing and supercobalancing numbers for $D=T_{k}$, where $k$ is any arbitrary positive integer. We also study some identities involving these numbers.

\section{Main results}

We start this section by showing that there exist at least two classes of $T_{k}$-superbalancing and $T_{k}$-subcobalancing numbers and at least one class of $T_{k}$-subbalancing and $T_{k}$-supercobalancing numbers for every positive integer $k$.

Theorem 2.1. For $k \geq 1$, the values of $x$ satisfying the Diophantine equation

$$
1+2+\cdots+w=(x+1)+(x+2)+\cdots+(x+r)+D,
$$

where $D= \pm T_{k}, w \in\{x-1, x\}$ may partition in multiple classes and the common classes of solutions are given by

(a) $k C_{l}+(2 k-1) B_{l}, k C_{l}-(2 k-1) B_{l} ; l \geqslant 1$ when $(w, D)=\left(x-1, T_{k}\right)$,

(b) $(2 k+1) B_{l} ; l \geqslant 1$ when $(w, D)=\left(x-1,-T_{k}\right)$,

(c) $(2 k+1) b_{l}+k ; l \geqslant 1$ when $(w, D)=\left(x, T_{k}\right)$,

(d) $\frac{1}{2}\left[(4 k-1) B_{l}+B_{l-1}-1\right], \frac{1}{2}\left[(4 k-1) B_{l}+B_{l+1}-1\right] ; l \geqslant 1$ when $(w, D)=\left(x,-T_{k}\right)$. 
Proof. (a) By virtue of equation (4), $8 x^{2}-8 T_{k}+1$ is perfect square. The congruence

$$
(2 k-1)^{2} x^{2} \equiv k^{2}\left(8 x^{2}-8 T_{k}+1\right)\left(\bmod 8 T_{k}-1\right)
$$

is equivalent to

$$
(2 k-1)^{2} x^{2} \equiv k^{2}\left(8 x^{2}-4 k^{2}-4 k+1\right)\left(\bmod 4 k^{2}+4 k-1\right)
$$

and is implied by

$$
(2 k-1) x \equiv \pm k \sqrt{8 x^{2}-4 k^{2}-4 k+1}\left(\bmod 4 k^{2}+4 k-1\right)
$$

and any solution of the latter congruence is a solution of the former and is a $T_{k}$-superbalancing number. In view of the latter congruence

$$
\frac{(2 k-1) x+k \sqrt{8 x^{2}-4 k^{2}-4 k+1}}{4 k^{2}+4 k-1} \text { or } \frac{(2 k-1) x-k \sqrt{8 x^{2}-4 k^{2}-4 k+1}}{4 k^{2}+4 k-1}
$$

is a natural number. Since

$$
8\left[\frac{(2 k-1) x \pm k \sqrt{8 x^{2}-4 k^{2}-4 k+1}}{4 k^{2}+4 k-1}\right]^{2}+1=\left[\frac{8 k x \pm(2 k-1) \sqrt{8 x^{2}-4 k^{2}-4 k+1}}{4 k^{2}+4 k-1}\right]^{2},
$$

it follows that either

$$
\frac{8 k x+(2 k-1) \sqrt{8 x^{2}-4 k^{2}-4 k+1}}{4 k^{2}+4 k-1} \text { or } \frac{8 k x-(2 k-1) \sqrt{8 x^{2}-4 k^{2}-4 k+1}}{4 k^{2}+4 k-1}
$$

is a Lucas-balancing number [12]. Letting

$$
C=\frac{8 k x \pm(2 k-1) \sqrt{8 x^{2}-4 k^{2}-4 k+1}}{4 k^{2}+4 k-1}
$$

we get

$$
\left[8 k x-\left(4 k^{2}+4 k-1\right) C\right]^{2}=(2 k-1)^{2}\left(8 x^{2}-4 k^{2}-4 k+1\right),
$$

which, on rearrangement, results in the quadratic equation

$$
8 x^{2}-16 C k x+\left(4 k^{2}+4 k-1\right) C^{2}+\left(4 k^{2}+4 k-1\right)=0,
$$

and the solutions are

$$
x=k C \pm(2 k-1) B .
$$

We further observe that

$$
8[k C \pm(2 k-1) B]^{2}-4 k^{2}-4 k+1=[(2 k-1) C \pm 8 B k]^{2} .
$$

Thus, two classes of $T_{k}$-superbalancing numbers are $k C_{l}+(2 k-1) B_{l}$ and $k C_{l}-(2 k-1) B_{l}$ for $l \geqslant 1$.

The proofs of (b), (c) and (d) are similar and we prefer to omit these. 
In the previous theorem, for each positive integer $k$, we ascertained the existence of at least one class of $T_{k}$-supercobbalancing and $T_{k}$-subbalancing numbers and at least two classes of $T_{k}$-superbbalancing and $T_{k}$-subcobalancing numbers. In the following theorem, we explore the exact number of such classes when $k$ and $D$ are suitably restricted.

Theorem 2.2. For $k \geq 1$, the classes of solutions of (4) given in Theorem 2.1 are exact, if the following holds:

(a) $4 k^{2}+4 k-1$ is a prime when $(w, D) \in\left\{\left(x-1, T_{k}\right),\left(x,-T_{k}\right)\right\}$,

(b) $p \equiv \pm 3(\bmod 8)$ for all $p \mid(2 k+1)$ when $(w, D) \in\left\{\left(x-1,-T_{k}\right),\left(x, T_{k}\right)\right\}$.

Proof. We prove this theorem only for the case $(w, D)=\left(x-1, T_{k}\right)$ as the other cases can be handled in a similar fashion.

If $(w, D)=\left(x-1, T_{k}\right)$, then it follows from (4) that $x$ is a $T_{k}$-superbalancing number and thus, $8 x^{2}-8 T_{k}+1$ is a perfect square. Let

$$
y^{2}=8 x^{2}-8 T_{k}+1=8 x^{2}-\left(4 k^{2}+4 k-1\right)
$$

and so

$$
y^{2} \equiv 8 x^{2} \quad\left(\bmod 4 k^{2}+4 k-1\right) .
$$

Now, (5) is solvable if and only if $\left(\frac{8 x^{2}}{4 k^{2}+4 k-1}\right)=1$ (see [9, p. 193]). Since $4 k^{2}+4 k-1$ is a prime congruent to $\pm 1(\bmod 8)$, we have

$$
\left(\frac{8 x^{2}}{4 k^{2}+4 k-1}\right)=\left(\frac{2}{4 k^{2}+4 k-1}\right)=1
$$

(see [9, p. 184]). So (5) is solvable and there are exactly two classes of $T_{k}$-superbalancing numbers (see [9, p. 156]), which can be derived from Theorem 2.1. Moreover, since $C_{n}=3 B_{n}-B_{n-1}=B_{n+1}-3 B_{n}$, we have

$$
k C_{l}-(2 k-1) B_{l}=(k+1) B_{l}-k B_{l-1}, \quad k C_{l}+(2 k-1) B_{l}=k B_{l+1}-(k+1) B_{l} .
$$

Further,

$$
8\left[(k+1) B_{l}-k B_{l-1}\right]^{2}-\left(4 k^{2}+4 k-1\right)=\left[(k+1) C_{l}-k C_{l-1}\right]^{2}
$$

and

$$
8\left[k B_{l+1}-(k+1) B_{l}\right]^{2}-\left(4 k^{2}+4 k-1\right)=\left[k C_{l+1}-(k+1) C_{l}\right]^{2}
$$

validates the two classes of $T_{k}$-superbalancing number.

\subsection{Relationships with triangular, square triangular, balancing and related numbers}

Here, we establish some relationship of the common classes of solution corresponding to $T_{k}$-superbalancing, $T_{k}$-subbalancing, $T_{k}$-supercobalancing and $T_{k}$-subcobalancing numbers with balancing, cobalancing, Lucas-balancing, Lucas-cobalancing, triangular and square triangular numbers.

For the sake of simplicity, we first denote the common class of solutions corresponding to these numbers as follows: 
$T_{k}$-subbalancing numbers $: T_{k} B_{n}^{*}$

$T_{k}$-superbalancing numbers $: T_{k} B_{n}^{* *}$

$T_{k}$-subcobalancing numbers $: T_{k} b_{n}^{*}$

$T_{k}$-supercobalancing numbers $: T_{k} b_{n}^{* *}$
$T_{k}$-Lucas - subbalancing numbers $: T_{k} C_{n}^{*}$

$T_{k}$-Lucas - superbalancing numbers $: T_{k} C_{n}^{* *}$

$T_{k}$-Lucas - subcobalancing numbers $: T_{k} c_{n}^{*}$

$T_{k}$-Lucas - supercobalancing numbers : $T_{k} c_{n}^{* *}$

Further,

$$
\begin{array}{ll}
T_{k} C_{n}^{*}=\sqrt{8\left(T_{k} B_{n}^{*}\right)^{2}+4 k^{2}+4 k+1} & T_{k} C_{n}^{* *}=\sqrt{8\left(T_{k} B_{n}^{* *}\right)^{2}-4 k^{2}-4 k+1} \\
T_{k} c_{n}^{*}=\sqrt{8\left(T_{k} b_{n}^{*}\right)^{2}+8 \cdot T_{k} b_{n}^{*}+4 k^{2}+4 k+1} & T_{k} c_{n}^{* *}=\sqrt{8\left(T_{k} B_{n}^{* *}\right)^{2}+8 \cdot T_{k} b_{n}^{* *}-4 k^{2}-4 k+1}
\end{array}
$$

\subsection{Relations with balancing and related numbers}

In the following three theorems, we represent subbalancing and superbalancing numbers in terms of balancing, cobalancing, Lucas-balancing and Lucas-cobalancing numbers.

Theorem 2.3. For every positive integer $k$, we have
(a) $T_{k} B_{n}^{*}=(2 k+1) B_{n}$
(c) $T_{k} b_{n}^{* *}=(2 k+1) b_{n}+k$
(b) $T_{k} C_{n}^{*}=(2 k+1) C_{n}$
(d) $T_{k} c_{n}^{* *}=(2 k+1) c_{n}$

Proof. The proofs of $(a)$ and (c) follows from Theorem $2.1-(b)$ and (c), respectively. Further, since $T_{k} C_{n}^{*}=\sqrt{8\left(T_{k} B_{n}^{*}\right)^{2}+(2 k+1)^{2}}$ and $T_{k} c_{n}^{* *}=\sqrt{8\left(T_{k} b_{n}^{* *}\right)^{2}+8 T_{k} b_{n}^{* *}-4 k^{2}-4 k+1}$, the representations in $(b)$ and $(d)$ immediately follows from $(a)$ and $(c)$, respectively.

Theorem 2.4. For every positive integer $k$, we have

(a) $T_{k} B_{2 n-1}^{* *}=k B_{n}-(k+1) B_{n-1}=k C_{n-1}+(2 k-1) B_{n-1}$

(b) $T_{k} B_{2 n}^{* *}=(k+1) B_{n}-k B_{n-1}=k C_{n}-(2 k-1) B_{n}=(k+1) C_{n-1}+(2 k+3) B_{n-1}$

(c) $T_{k} C_{2 n-1}^{* *}=k C_{n}-(k+1) C_{n-1}=(8 k+2) B_{n}-(2 k+1) C_{n}=(8 k) B_{n-1}+(2 k-1) C_{n-1}$

(d) $T_{k} C_{2 n}^{* *}=(k+1) C_{n}-k C_{n-1}=8 k B_{n}-(2 k-1) C_{n}=8(k+1) B_{n-1}+(2 k+3) C_{n-1}$

Theorem 2.5. For every positive integer $k$, we have

(a) $T_{k} b_{2 n-1}^{*}=\frac{1}{2}\left[(4 k-1) B_{n}+B_{n+1}-1\right]=k b_{n+1}-(k+1) b_{n}-1$

(b) $T_{k} b_{2 n}^{*}=\frac{1}{2}\left[(4 k-1) B_{n}+B_{n-1}-1\right]=(k+1) b_{n+1}-k b_{n}$

(c) $T_{k} c_{2 n-1}^{*}=(6 k-1) B_{n}-(2 k+1) B_{n-1}=c_{n+1}-2 c_{n}+2(k-1)\left(b_{n+1}+b_{n}+1\right)$

(d) $T_{k} c_{2 n}^{*}=(2 k+1) B_{n+1}-(6 k-1) B_{n}=c_{n+2}-4 c_{n+1}+2(k-1)\left(b_{n+1}+b_{n}+1\right)$

The proofs of Theorem 2.4 and 2.5 are similar to that of Theorem 2.3. Further, with $k=1$, some of the representations from Theorems $2.3-2.5$ can be seen in [16]. 
Just like cobalancing numbers, the $T_{k}$-supercobalancing and $T_{k}$-subcobalancing numbers satisfy non-linear recurrence relations, where as the recurrence relation for others are linear.

Theorem 2.6. For every positive integer $k$,

(i) the recurrence $x_{n+1}=6 x_{n}-x_{n-1}$ is satisfied by $T_{k} B_{n}^{*}, T_{k} C_{n}^{*}$ and $T_{k} c_{n}^{* *}$ with initial terms $T_{k} B_{0}^{*}=0, T_{k} B_{1}^{*}=T_{k} C_{0}^{*}=T_{k} c_{1}^{* *}=2 k+1, T_{k} C_{1}^{*}=3(2 k+1), T_{k} c_{0}^{* *}=-(2 k+1)$,

(ii) the recurrence $x_{n+2}=6 x_{n}-x_{n-2}$ is satisfied by $T_{k} B_{n}^{* *}, T_{k} C_{n}^{* *}$ and $T_{k} c_{n}^{*}$ with initial terms $T_{k} B_{0}^{* *}=T_{k} B_{1}^{* *}=k, T_{k} B_{2}^{* *}=k+1, T_{k} B_{3}^{* *}=5 k-1, T_{k} C_{0}^{* *}=-2 k+1, T_{k} C_{1}^{* *}=$ $2 k+1, T_{k} C_{2}^{* *}=2 k+3, T_{k} C_{3}^{* *}=14 k-3, T_{k} c_{0}^{*}=2 k+1, T_{k} c_{1}^{*}=6 k-1, T_{k} c_{2}^{*}=$ $6 k+7, T_{k} c_{3}^{*}=34 k-7$,

(iii) $T_{k} b_{n}^{*}$ and $T_{k} b_{n}^{* *}$ satisfy the recurrence $x_{n+2}=6 x_{n}-x_{n-2}+2$ and $x_{n+1}=6 x_{n}-x_{n-1}+2$ respectively and the initial terms are $T_{k} b_{0}^{*}=0, T_{k} b_{1}^{*}=2 k-1, T_{k} b_{2}^{*}=2 k+2, T_{k} b_{3}^{*}=$ $12 k-3, T_{k} b_{0}^{* *}=T_{k} b_{1}^{* *}=k$.

In view of Theorem 2.3, one can easily find the following Binet forms.

$$
\begin{gathered}
T_{k} B_{n}^{*}=(2 k+1)\left(\frac{\alpha^{2 n}-\beta^{2 n}}{4 \sqrt{2}}\right), \quad T_{k} C_{n}^{*}=(2 k+1)\left(\frac{\alpha^{2 n}+\beta^{2 n}}{2}\right), \\
T_{k} b_{n}^{* *}=(2 k+1)\left(\frac{\alpha^{2 n-1}-\beta^{2 n-1}}{4 \sqrt{2}}\right)-\frac{1}{2}, \quad T_{k} c_{n}^{* *}=(2 k+1)\left(\frac{\alpha^{2 n-1}+\beta^{2 n-1}}{2}\right),
\end{gathered}
$$

where $\alpha=1+\sqrt{2}$ and $\beta=1-\sqrt{2}$.

\subsection{Relations with triangular and square triangular numbers}

Let $S_{n}$ denote the $n$-th square triangular number and hence

$$
S_{n}=s_{n}^{2}=\frac{t_{n}\left(t_{n}+1\right)}{2} .
$$

Note that $s_{n}=B_{n}$. The Binet formulas of $S_{n}, s_{n}$ and $t_{n}$ are given by

$$
S_{n}=\frac{\alpha^{4 n}+\beta^{4 n}-2}{32}, \quad s_{n}=\frac{\alpha^{2 n}-\beta^{2 n}}{4 \sqrt{2}}, \quad t_{n}=\frac{\alpha^{2 n}+\beta^{2 n}-2}{4}
$$

for $n \geq 1$ and $S_{0}=s_{0}=t_{0}=0($ see $[1,11,21])$.

In [11], Özkoç et al. derived some new results on triangular and square triangular numbers involving generalized Pell numbers. In this subsection, we present the following theorems which are similar to Theorems 2.1 to 2.7 in [21].

Theorem 2.7. Let $n$ be any positive integer. Then, for triangular numbers, we have

1. $T_{\frac{T_{k} B_{n}^{*}+T_{k} B_{n-1}^{*}-(2 k+1)}{4 k+2}}=\frac{\left[T_{k} b_{2 n-1}^{*}-T_{k} b_{2 n-2}^{*}-(2 k-1)\right]\left[T_{k} b_{2 n-1}^{*}-T_{k} b_{2 n-2}^{*}+(2 k-1)\right]}{4(2 k-1)^{2}}$

2. $\frac{T_{7 T_{k} B_{n}^{*}-T_{k} B_{n-1}^{*}-(2 k+1)}}{4 k+2}=\frac{(4 k-2) T_{k} B_{2 n+1}^{*}+(2 k+1)\left(T_{k} b_{4 n+1}^{*}-T_{k} b_{4 n}^{*}\right)-3\left(4 k^{2}-1\right)}{16\left(4 k^{2}-1\right)}$ 
3. $\frac{T_{T_{k} B_{2 n+1}^{* *}-T_{k} B_{2 n}^{* *}+T_{k} B_{2 n-1}^{* *}-T_{k} B_{2 n-2}^{* *}-2}}{4}$

$$
=\frac{2(2 k-1)^{2}\left(T_{k} b_{n}^{* *}-k\right)\left(T_{k} b_{n}^{* *}+k+1\right)+k(k-1)(2 k+1)^{2}}{2(2 k+1)^{2}}
$$

4. $\frac{T_{7 T_{k} B_{2 n+1}^{* *}-7 T_{k} B_{2 n}^{* *}-T_{k} B_{2 n-1}^{* *}+T_{k} B_{2 n-2}^{* *}-2}}{4}$

$$
=\frac{\left[\left(4 k^{2}-1\right)\left(T_{k} B_{4 n+3}^{* *}-T_{k} B_{4 n+2}^{* *}\right)+2(2 k-1)^{2} T_{k} b_{2 n+1}^{* *}-(4 k-2)\left(2 k^{2}-k+1\right)-4\right]}{16(2 k+1)} .
$$

Proof. Using the Binet formulas $B_{n}=\left(\frac{\alpha^{2 n}-\beta^{2 n}}{4 \sqrt{2}}\right)$ and $T_{k} B_{n}^{*}=(2 k+1) B_{n}$ with $\alpha \beta=-1$, we have

$$
\begin{aligned}
T_{\frac{T_{k} B_{n}^{*}+T_{k} B_{n-1}^{*}-(2 k+1)}{4 k+2}} & =\frac{\left(\frac{T_{k} B_{n}^{*}+T_{k} B_{n-1}^{*}-(2 k+1)}{4 k+2}\right)\left(\frac{T_{k} B_{n}^{*}+T_{k} B_{n-1}^{*}-(2 k+1)}{4 k+2}+1\right)}{2} \\
& =\frac{\left(T_{k} B_{n}^{*}+T_{k} B_{n-1}^{*}\right)^{2}-(2 k+1)^{2}}{8(2 k+1)^{2}} \\
& =\frac{\left(B_{n}+B_{n-1}\right)^{2}-1}{8} \\
& =\frac{\left(\alpha^{2 n}-\beta^{2 n}+\alpha^{2 n-2}-\beta^{2 n-2}\right)^{2}-32}{256} \\
& =\frac{\left[\left(\alpha^{2 n-1}\left(\alpha+\alpha^{-1}\right)-\beta^{2 n-1}\left(\beta+\beta^{-1}\right)\right]^{2}-32\right.}{256} \\
& =\frac{(\alpha-\beta)^{2}\left(\alpha^{2 n-1}+\beta^{2 n-1}\right)^{2}-32}{256} \\
& =\frac{\left(\alpha^{2 n-1}+\beta^{2 n-1}\right)^{2}-4}{32} \\
& =\frac{\left(\alpha^{2 n-1}-\beta^{2 n-1}\right)^{2}-8}{32} \\
& =\left(\frac{\alpha^{2 n-1}-\beta^{2 n-1}}{4 \sqrt{2}}\right)^{2}-\frac{1}{4} \\
& =\left(\frac{\alpha^{2 n-1}-\beta^{2 n-1}}{4 \sqrt{2}}-\frac{1}{2}\right)\left(\frac{\alpha^{2 n-1}-\beta^{2 n-1}}{4 \sqrt{2}}+\frac{1}{2}\right) \\
& =\left(\frac{T_{k} b_{2 n-1}^{*}-T_{k} b_{2 n-2}^{*}}{4 k-2}-\frac{1}{2}\right)\left(\frac{T_{k} b_{2 n-1}^{*}-T_{k} b_{2 n-2}^{*}}{4 k-2}+\frac{1}{2}\right) \\
& =\frac{\left[T_{k} b_{2 n-1}^{*}-T_{k} b_{2 n-2}^{*}-(2 k-1)\right]\left[T_{k} b_{2 n-1}^{*}-T_{k} b_{2 n-2}^{*}+(2 k-1)\right]}{4(2 k-1)^{2}} .
\end{aligned}
$$

This proves 1 . The proofs of 2.- 4 . follow similarly and hence, they are omitted.

Theorem 2.8. Let $n$ be any positive integer. Then, for triangular numbers, we have

1. $\frac{T_{T_{k} B_{n}^{*}+T_{k} B_{n-1}^{*}-(2 k+1)}}{4 k+2}=\frac{\left(T_{k} b_{n}^{* *}-k\right)\left(T_{k} b_{n}^{* *}+k+1\right)}{(2 k+1)^{2}}$

2. $\frac{T_{7 T_{k} B_{n}^{*}-T_{k} B_{n-1}^{*}-(2 k+1)}}{4 k+2}=\frac{T_{k} B_{2 n+1}^{*}+T_{k} b_{2 n+1}^{* *}-(3 k+1)}{8(2 k+1)}$

3. $\frac{T_{T_{k} B_{2 n+1}^{* *}-T_{k} B_{2 n}^{* *}+T_{k} B_{2 n-1}^{* *}-T_{k} B_{2 n-2}^{* *}-2}}{4}=\left[2(2 k-1)^{2}\left(2 T_{k} b_{2 n-2}^{*}-T_{k} b_{2 n-3}^{*}+(k-1) C_{n-1}-k\right)\right.$

$$
\left.\left(2 T_{k} b_{2 n-2}^{*}-T_{k} b_{2 n-3}^{*}+(k-1) C_{n-1}+k+1\right)+k(k-1)(2 k+1)^{2}\right] /\left[2(2 k+1)^{2}\right]
$$


4. $\frac{T_{7 T_{k} B_{2 n+1}^{* *}-7 T_{k} B_{2 n}^{* *}-T_{k} B_{2 n-1}^{* *}+T_{k} B_{2 n-2}^{* *}-2}}{4}=\left[3\left(4 k^{2}-1\right)\left(T_{k} B_{4 n+3}^{* *}-T_{k} B_{4 n+2}^{* *}\right)-12+2(2 k-1)^{2}\right.$
$\left.\left[(2 k+1)\left(2 T_{k} b_{4 n}^{*}-T_{k} b_{4 n-1}^{*}\right)-(k-1)\left(2 B_{2 n}-1\right)\right]-(12 k-6)\left(2 k^{2}-k+1\right)\right] /[48(2 k+1)]$.

Proof. 1. In view of the Theorem 2.7, it is easy to show that

$$
\begin{aligned}
& T_{\frac{T_{k} B_{n}^{*}+T_{k} B_{n-1}^{*}-(2 k+1)}{4 k+2}} \\
& =\left(\frac{\alpha^{2 n-1}-\beta^{2 n-1}}{4 \sqrt{2}}-\frac{1}{2}\right)\left(\frac{\alpha^{2 n-1}-\beta^{2 n-1}}{4 \sqrt{2}}+\frac{1}{2}\right) \\
& =\frac{\left[(2 k+1)\left(\frac{\alpha^{2 n-1}-\beta^{2 n-1}}{4 \sqrt{2}}\right)-\frac{1}{2}-k\right]\left[(2 k+1)\left(\frac{\alpha^{2 n-1}-\beta^{2 n-1}}{4 \sqrt{2}}\right)-\frac{1}{2}+(k+1)\right]}{(2 k+1)^{2}} \\
& =\frac{\left(T_{k} b_{n}^{* *}-k\right)\left(T_{k} b_{n}^{* *}+k+1\right)}{(2 k+1)^{2}} .
\end{aligned}
$$

The proofs of 2.- 4. follows similarly and hence, they are omitted.

Theorem 2.9. The general terms of $S_{n}, s_{n}$ and $t_{n}$ for $n \geq 1$ are given by

$$
S_{n}=\left(\frac{T_{k} b_{2 n+1}^{*}-T_{k} b_{2 n}^{*}-T_{k} b_{2 n-1}^{*}+T_{k} b_{2 n-2}^{*}}{4(2 k-1)}\right)^{2}, s_{n}=\frac{T_{k} B_{n}^{*}}{2 k+1}, t_{n}=\frac{T_{k} C_{n}^{*}-(2 k+1)}{2(2 k+1)}
$$

or

$$
S_{n}=\left(\frac{T_{k} b_{n+1}^{* *}-T_{k} b_{n}^{* *}}{2(2 k+1)}\right)^{2}, s_{n}=\frac{T_{k} B_{2 n+1}^{* *}-T_{k} B_{2 n}^{* *}}{2(2 k-1)}, t_{n}=\frac{T_{k} C_{2 n+1}^{* *}-T_{k} C_{2 n}^{* *}-2(2 k-1)}{4(2 k-1)} .
$$

Proof. Since $S_{n}=s_{n}^{2}=B_{n}^{2}$ and

$$
\begin{aligned}
B_{n}= & \frac{(7 k-3 k-2)\left(b_{n+1}-b_{n}\right)}{4(2 k-1)} \\
= & \frac{k\left(b_{n+2}-b_{n-1}\right)-(3 k+2)\left(b_{n+1}-b_{n}\right)}{4(2 k-1)} \\
= & \frac{1}{4(2 k-1)}\left(\left[k b_{n+2}-(k+1) b_{n+1}-1\right]-\left[(k+1) b_{n+1}-k b_{n}\right]\right. \\
& \left.-\left[k b_{n+1}-(k+1) b_{n}-1\right]+\left[(k+1) b_{n}-k b_{n-1}\right]\right) \\
= & \left.\frac{T_{k} b_{2 n+1}^{*}-T_{k} b_{2 n}^{*}-T_{k} b_{2 n-1}^{*}+T_{k} b_{2 n-2}^{*}}{4(2 k-1)} \quad \text { (see Theorem } 2.5\right),
\end{aligned}
$$

the first identity follows. The second identity can be proved using Theorem $2.3-(a)$ and the third identity follows from

$$
t_{n}=\frac{\alpha^{2 n}+\beta^{2 n}-2}{4}=\frac{C_{n}-1}{2}=\frac{(2 k+1) C_{n}-(2 k+1)}{2(2 k+1)} .
$$

Other three identities can be proved in a similar fashion. 
Theorem 2.10. The general terms of $S_{n}$ for $n \geq 1$ is given by

1. $S_{n}=\left(\frac{-2 T_{k} B_{n-1}^{*}+T_{k} C_{n}^{*}-T_{k} C_{n-1}^{*}}{2(2 k+1)}\right)^{2}$

2. $S_{n}=\frac{\left[2 T_{k} B_{n-1}^{*}+T_{k} C_{n-1}^{*}-(2 k+1)\right]\left[4 T_{k} B_{n}^{*}+2 T_{k} B_{n-1}^{*}+T_{k} C_{n-1}^{*}+(2 k+1)\right]+4(2 k+1) T_{k} B_{n}^{*}}{4(2 k+1)^{2}}$

3. $S_{n}=\left(\frac{-2 T_{k} B_{2 n-1}^{* *}+2 T_{k} B_{2 n-2}^{* *}+T_{k} C_{2 n+1}^{* *}-T_{k} C_{2 n}^{* *}-T_{k} C_{2 n-1}^{* *}+T_{k} C_{2 n-2}^{* *}}{4(2 k-1)}\right)^{2}$

4. $S_{n}=\left[\left(2 T_{k} B_{2 n-1}^{* *}-2 T_{k} B_{2 n-2}^{* *}+T_{k} C_{2 n-1}^{* *}-T_{k} C_{2 n-2}^{* *}-4 k+2\right)\left(4 T_{k} B_{2 n+1}^{* *}-4 T_{k} B_{2 n}^{* *}+\right.\right.$ $\left.\left.2 T_{k} B_{2 n-1}^{* *}-2 T_{k} B_{2 n-2}^{* *}+T_{k} C_{2 n-1}^{* *}-T_{k} C_{2 n-2}^{* *}+4 k-2\right)+8 T_{k} B_{2 n+1}^{* *}-8 T_{k} B_{2 n}^{* *}\right] /\left[16(2 k-1)^{2}\right]$.

Proof. Proof of this theorem is similar to that of Theorem 2.7 and hence, we prefer to omit it.

Theorem 2.11. For $n \geq 1$, the general terms of $T_{k}$-subbalancing and $T_{k}$-subcobalancing numbers are

$$
\begin{aligned}
T_{k} B_{n}^{*} & =(2 k+1) s_{n} \\
T_{k} b_{2 n}^{*} & =-2 s_{n+1}+(2 k-1) s_{n}+2 t_{n+1}-t_{n} \\
T_{k} b_{2 n-1}^{*} & =(2 k-6) s_{n}+s_{n-1}+4 t_{n}-t_{n-1}+1 \\
T_{k} C_{n}^{*} & =(2 k+1)\left(2 t_{n}+1\right) \\
T_{k} c_{2 n}^{*} & =(2 k+1) s_{n+1}-(6 k-1) s_{n} \\
T_{k} c_{2 n-1}^{*} & =(6 k-1) s_{n}-(2 k+1) s_{n-1}
\end{aligned}
$$

and the general terms of $T_{k}$-superbalancing and $T_{k}$-supercobalancing numbers are

$$
\begin{aligned}
T_{k} B_{2 n}^{* *} & =-(2 k-1) s_{n}+k\left(2 t_{n}+1\right) \\
T_{k} B_{2 n-1}^{* *} & =(2 k-1) s_{n-1}+k\left(2 t_{n-1}+1\right) \\
T_{k} b_{n}^{* *} & =(2 k+1)\left(t_{n}-s_{n}\right)+k \\
T_{k} C_{2 n}^{* *} & =8 k s_{n}-(2 k-1)\left(2 t_{n}+1\right) \\
T_{k} C_{2 n-1}^{* *} & =8 k s_{n-1}+(2 k-1)\left(2 t_{n}+1\right) \\
T_{k} c_{n}^{* *} & =(2 k+1)\left(s_{n}+s_{n-1}\right) .
\end{aligned}
$$

Proof. Proceeding as in Theorem 2.7, it is easy to obtain these representations. Hence, we prefer to omit the proof.

Theorem 2.12. For any natural number $n \geq 1$, the sums of first $n$-terms of $S_{n}, s_{n}, t_{n}$ are

$$
\begin{aligned}
\sum_{i=1}^{n} S_{i} & =\frac{33\left(T_{k} B_{n}^{*}\right)^{2}-\left(T_{k} B_{n-1}^{*}\right)^{2}-(2 k+1)^{2}(2 n-1)}{32(2 k+1)^{2}} \\
\sum_{i=1}^{n} s_{i} & =\frac{5 T_{k} B_{n}^{*}-T_{k} B_{n-1}^{*}-(2 k+1)}{4(2 k+1)} \\
\sum_{i=1}^{n} t_{i} & =\frac{7 T_{k} B_{n}^{*}-T_{k} B_{n-1}^{*}-(2 k+1)(2 n+1)}{4(2 k+1)}
\end{aligned}
$$


or

$$
\begin{aligned}
\sum_{i=1}^{n} S_{i} & =\frac{824\left(T_{k} b_{n}^{* *}\right)^{2}-328 T_{k} b_{n}^{* *} T_{k} b_{n-1}^{* *}+660 T_{k} b_{n}^{* *}+132+32\left(T_{k} b_{n-1}^{* *}\right)^{2}-132 T_{k} b_{n-1}^{* *}-(2 k+1)^{2}(8 n-4)}{128(2 k+1)^{2}}, \\
\sum_{i=1}^{n} s_{i} & =\frac{5\left(T_{k} B_{2 n+1}^{* *}-T_{k} B_{2 n}^{* *}\right)-\left(T_{k} B_{2 n-1}^{* *}-T_{k} B_{2 n-2}^{* *}\right)-(4 k-2)}{8(2 k-1)} \\
\sum_{i=1}^{n} t_{i} & =\frac{7\left(T_{k} B_{2 n+1}^{* *}-T_{k} B_{2 n}^{* *}\right)-\left(T_{k} B_{2 n-1}^{* *}-T_{k} B_{2 n-2}^{* *}\right)-(4 k-2)(2 n+1)}{8(2 k-1)} .
\end{aligned}
$$

Proof. Since $S_{n}=s_{n}^{2}$ and $\sum_{i=1}^{n} S_{i}=\frac{33 S_{n}-S_{n-1}-2 n+1}{32}$ (see [21, p. 116]), using Theorem 2.3, it is easy to see that

$$
\begin{aligned}
\sum_{i=1}^{n} S_{i} & =\frac{33(2 k+1)^{2} s_{n}^{2}-(2 k+1)^{2} s_{n-1}^{2}-(2 k+1)^{2}(2 n-1)}{32(2 k+1)^{2}} \\
& =\frac{33\left(T_{k} B_{n}^{*}\right)^{2}-\left(T_{k} B_{n-1}^{*}\right)^{2}-(2 k+1)^{2}(2 n-1)}{32(2 k+1)^{2}}
\end{aligned}
$$

The other summation results follow similarly. Hence, we omit their proofs.

Theorem 2.13. Let $n \geq 1$ be any natural number, then
1. $S_{n}=\frac{T_{T_{k} B_{n+1}^{*}-T_{k} B_{n-1}^{*}-2(2 k+1)}}{4(2 k+1)}$
2. $S_{n}=\frac{T_{(2 k-2) T_{k} B_{n-1}^{*}-(2 k-4) T_{k} B_{n}^{*}+(2 k+1)\left(T_{k} b_{2 n-1}^{*}-T_{k} b_{2 n-2}^{*}-1\right)}}{2(2 k+1)}$
3. $S_{n}=\frac{T_{T_{k} B_{2 n+3}^{* *}-T_{k} B_{2 n+2}^{* *}-T_{k} B_{2 n-1}^{* *}+T_{k} B_{2 n-2}^{* *}-4(2 k-1)}}{8(2 k-1)}$
4. $S_{n}=\frac{T_{(2 k+1)\left(T_{k} B_{2 n+1}^{* *}-T_{k} B_{2 n}^{* *}\right)+(4 k-2) T_{k} b_{n}^{* *}-2 k(2 k-1)}}{2\left(4 k^{2}-1\right)}$.

Proof. In view of Theorem 2.3, we have

$$
\begin{aligned}
\frac{T_{T_{k} B_{n+1}^{*}-T_{k} B_{n-1}^{*}-2(2 k+1)}^{4(2 k+1)}}{2} & =\frac{\left(T_{k} B_{n+1}^{*}-T_{k} B_{n-1}^{*}\right)^{2}-4(2 k+1)^{2}}{32(2 k+1)^{2}} \\
& =\frac{\left(B_{n+1}-B_{n-1}\right)^{2}-4}{32} \\
& =\frac{\left(\frac{\alpha^{2 n+2}-\beta^{2 n+2}}{4 \sqrt{2}}-\frac{\alpha^{2 n-2}-\beta^{2 n-2}}{4 \sqrt{2}}\right)^{2}-4}{32} \\
& =\frac{\left[\alpha^{2 n}\left(\alpha^{2}-\alpha^{-2}\right)-\beta^{2 n}\left(\beta^{2}-\beta^{-2}\right)\right]^{2}-128}{1024} \\
& =\frac{\left(\alpha^{2}-\beta^{2}\right)^{2}\left(\alpha^{2 n}+\beta^{2 n}\right)^{2}-128}{1024} \\
& =\frac{\alpha^{4 n}+\beta^{4 n}-2}{32}=S_{n} .
\end{aligned}
$$

This completes the proof 1 . The proofs of 2.-4. are similar to that of 1 . and hence, we omit them. 
Theorem 2.14. For any natural number $n \geq 1$, we have

1. $\sqrt{8\left(s_{n-1}+t_{n-1}\right)^{2}+8\left(s_{n-1}+t_{n-1}\right)+1}$

$$
=\frac{T_{k} B_{n}^{*}+T_{k} B_{n-1}^{*}}{(2 k+1)} \text { or } \frac{T_{k} B_{2 n+1}^{* *}-T_{k} B_{2 n}^{* *}+T_{k} B_{2 n-1}^{* *}-T_{k} B_{2 n-2}^{* *}}{2(2 k-1)}
$$

2. $\sqrt{S_{n}-t_{n}-2 s_{n}\left(s_{n-1}+t_{n-1}\right)}$

$$
=\frac{T_{k} b_{n}^{* *}-k}{2 k+1} \text { or } \frac{T_{k} b_{2 n-1}^{* *}-T_{k} b_{2 n-2}^{* *}-(2 k-1)}{2(2 k-1)}
$$

3. $\sqrt{\left(s_{n-1}+t_{n-1}\right)^{2}+t_{n}+2 s_{n}\left(s_{n-1}+t_{n-1}\right)}$

$$
\begin{aligned}
& =\frac{(2 k-1) T_{k} C_{n}^{*}-(2 k+1)\left(T_{k} b_{2 n-1}^{*}-T_{k} b_{2 n-2}^{*}\right)}{2\left(4 k^{2}-1\right)} \\
& \text { or } \frac{(2 k+1)\left(T_{k} C_{2 n+1}^{*}-T_{k} C_{2 n}^{*}\right)-4(2 k-1) T_{k} b_{n}^{* *}-(4 k-2)}{2\left(4 k^{2}-1\right)} \\
& \text { or } \frac{(4 k+2)\left(T_{k} C_{2 n+1}^{*}-T_{k} C_{2 n}^{*}\right)-(2 k-1)\left(T_{k} c_{n+1}^{* *}-3 T_{k} c_{n}^{* *}\right)}{4\left(4 k^{2}-1\right)}
\end{aligned}
$$

4. $\sqrt{\frac{s_{2 n}+s_{2 n-1}+t_{2 n-1}}{2}}=\frac{2 T_{k} B_{n}^{*}}{2 k+1}$ or $\frac{T_{k} B_{2 n+1}^{* *}-T_{k} B_{2 n}^{* *}}{2 k-1}$

5. $\sqrt{t_{2 n-1}}=\frac{T_{k} c_{n}^{* *}}{2 k+1}$ or $\frac{(2 k+1)\left(T_{k} c_{2 n-1}^{*}-T_{k} c_{2 n-2}^{*}\right)-(2 k-2) T_{k} b_{n+1}^{* *}+2 k(k-1)}{2(2 k+1)}$

$$
\text { or } \frac{4(2 k+1)\left(T_{k} c_{2 n-1}^{*}-T_{k} c_{2 n-2}^{*}\right)-(k-1)\left(T_{k} c_{n+1}^{* *}-3 T_{k} c_{n}^{* *}\right)+4(k-1)}{8(2 k+1)}
$$

and

$$
\sqrt{t_{2 n}+1}=\frac{T_{k} C_{n}^{*}}{2 k+1} \text { or } \frac{T_{k} C_{2 n+1}^{* *}-T_{k} C_{2 n}^{* *}}{4 k-2}
$$

Proof. 1. Since $\sqrt{8 b_{n}^{2}+8 b_{n}+1}=c_{n}=\frac{\alpha^{2 n-1}+\beta^{2 n-1}}{2}$ and

$$
s_{n-1}+t_{n-1}=\frac{\alpha^{2 n-2}-\beta^{2 n-2}}{4 \sqrt{2}}+\frac{\alpha^{2 n-2}+\beta^{2 n-2}-2}{4}=\frac{\alpha^{2 n-1}-\beta^{2 n-1}}{4 \sqrt{2}}-\frac{1}{2}=b_{n},
$$

it follows that

$$
\begin{aligned}
& \sqrt{8\left(s_{n-1}+t_{n-1}\right)^{2}+8\left(s_{n-1}+t_{n-1}\right)+1} \\
& =\frac{\alpha^{2 n-1}+\beta^{2 n-1}}{2} \\
& =\frac{\alpha^{2 n-1}(\alpha-\beta)+\beta^{2 n-1}(\alpha-\beta)}{2(\alpha-\beta)} \\
& =\frac{\alpha^{2 n-1}\left(\alpha+\alpha^{-1}\right)+\beta^{2 n-1}\left(-\beta^{-1}-\beta\right)}{4 \sqrt{2}} \\
& =\frac{\alpha^{2 n}-\beta^{2 n}+\frac{\alpha^{2 n-2}-\beta^{2 n-2}}{4 \sqrt{2}}}{4 \sqrt{2}} \\
& =\frac{T_{k} B_{n}^{*}+T_{k} B_{n-1}^{*}}{2 k+1} .
\end{aligned}
$$

The other cases can be proved similarly. 


\section{Concluding remark}

It can be seen from Theorem 2.2(b) that the Diophantine equations $8 x^{2}+(2 k+1)^{2}=y^{2}$ and $8 x^{2}+8 x-4 k^{2}-4 k+1=y^{2}$ results in exactly one class of solution when all the prime factors of $(2 k+1)$ are congruent to $\pm 3(\bmod 8)$. So, it is reasonable to look for the number of classes of solutions when $2 k+1$ involves prime factors other than $\pm 3(\bmod 8)$. After verifying several number of special cases we believe that the following conjecture is true.

Conjecture 3.1. For $k \geq 1$, let $p_{1}^{m_{1}} p_{2}^{m_{2}} \cdots p_{r}^{m_{r}} q_{1}^{n_{1}} q_{2}^{n_{2}} \cdots q_{s}^{n_{s}}$ be a canonical decomposition of $2 k+1$ with $m_{i}, n_{i} \in \mathbb{N}, p_{i}$ and $q_{j}$ be primes, $p_{i} \equiv \pm 3(\bmod 8)$ and $q_{j} \equiv \pm 1(\bmod 8)$ for $1 \leq i \leq r, 1 \leq j \leq s$. Then the solutions of the Diophantine equations $8 x^{2}+(2 k+1)^{2}=y^{2}$ and $8 x^{2}+8 x-4 k^{2}-4 k+1=y^{2}$ partition in $M=\prod_{j=1}^{s}\left(2 n_{j}+1\right)$ classes each.

\section{Acknowledgements}

The authors wish to express their sincere gratitude to the anonymous referees for their careful reading of the manuscript and for many valuable comments and suggestions which improved the presentation of the paper to a great extent.

\section{References}

[1] Behera, A., \& Panda, G. K. (1999). On the square roots of triangular numbers, Fib. Quart., 37 (2), 98-105.

[2] Dash, K. K., Ota, R. S., \& Dash, S. (2012). t-balancing Numbers, Int. J. Contemp. Math. Sciences, 7 (41), 1999-2012.

[3] Davala, R. K., \& Panda, G. K. (2016) Supercobalancing Numbers, Matematika, 32 (1), $31-42$.

[4] Davala, R. K., \& Panda, G. K. (2018). Subbalancing Numbers, Matematika, 34 (1), $163-$ 172.

[5] Gözeri, G. K., Özkoç, A., \& Tekcan, A. (2017) Some Algebraic Relations on Balancing Numbers, Utilitas Mathematica, 103, 217-236.

[6] Keskin, R., \& Karaatl, O. (2012). Some New Properties of Balancing Numbers and Square Triangular Numbers, J. Int. Seq., 15, Article 12.1.4.

[7] Kovács, T., Liptai, K., \& Olajos, P. (2010). On (a,b)-balancing Numbers, Publ. Math. Deb., 77 (3-4), 485-498.

[8] Liptai, K., Luca, F., Pinter, A., \& Szalay, L. (2009). On Generalized Balancing Numbers, Indag. Mathem., 20 (1), 87-100. 
[9] Mollin, R. A. (2004). Fundamental Number Theory with Applications, Boca Raton, CRC press, London.

[10] Olajos, P. (2010). Properties of Balancing, Cobalancing and Generalized Balancing Numbers, Annales Math. Info., 37, 125-138.

[11] Özkoç, A., Tekcan, A., \& Gözeri, G. K. (2017). Triangular and Square Triangular Numbers Involving Generalized Pell Numbers, Utilitas Mathematica, 102, 231-254.

[12] Panda, G.K. (2009). Some fascinating properties of balancing numbers, In Proc. of Eleventh Internat. Conference on Fibonacci Numbers and Their Applications, Cong. Numerantium, 194, 185-189.

[13] Panda, G. K., \& Panda, A. K. (2015). Almost balancing numbers, Journal of the Indian Math. Soc., 82 (3-4), 147-156.

[14] Panda, G. K., \& Ray, P. K. (2005). Cobalancing numbers and cobalancers, Int. J. Math. Math. Sci., 8, 1189-1200.

[15] Panda, G. K., \& Ray, P. K. (2011). Some Links of Balancing and Cobalancing Numbers with Pell and Associated Pell Numbers, Bull. Inst. Math., Acad. Sin., 6 (1), 41-72.

[16] Panda, A. K. (2017). Some Variants of the Balancing Sequences, Ph.D. thesis, National Institute of Technology Rourkela, India.

[17] Ray, P. K. (2009). Balancing and Cobalancing Numbers, Ph.D. thesis, Department of Mathematics, National Institute of Technology, Rourkela, India.

[18] Rout, S. S., \& Panda, G. K. (2015). k-gap balancing numbers, Period. Math. Hungar., 70 (1), 109-121.

[19] Tekcan, A., Tayat, M., \& Özbek, M. E. (2014). The Diophantine Equation $8 x^{2}-y^{2}+8 x(1+t)+(2 t+1)^{2}=0$ and $t$-Balancing Numbers, ISR Combinatorics, Article ID 897834.

[20] Tekcan, A., Özkoç, A., \& Özbek, M. E. (2016). Some Algebraic Relations on Integer Sequences Involving Oblong and Balancing Numbers, Ars Combinatoria, 128, 11-31.

[21] Tekcan, A. (2019). Almost balancing, triangular and square triangular numbers, Notes on Number Theory and Discrete Maths., 25 (1), 108-121. 